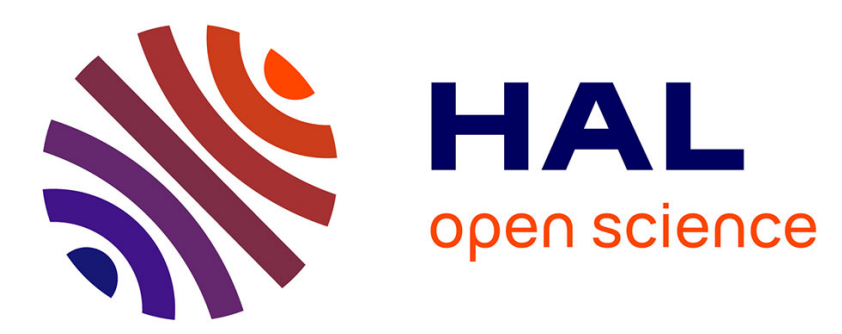

\title{
Artificial boundary conditions to compute correctors in linear elasticity
}

Virginie Bonnaillie-Noël, Delphine Brancherie, Marc Dambrine, Grégory Vial

\section{To cite this version:}

Virginie Bonnaillie-Noël, Delphine Brancherie, Marc Dambrine, Grégory Vial. Artificial boundary conditions to compute correctors in linear elasticity. Numerical Analysis and Applications, 2012, 5 (2), pp.129-135. 10.1134/S199542391202005X . hal-00951567

\section{HAL Id: hal-00951567 https://hal.science/hal-00951567}

Submitted on 25 Feb 2014

HAL is a multi-disciplinary open access archive for the deposit and dissemination of scientific research documents, whether they are published or not. The documents may come from teaching and research institutions in France or abroad, or from public or private research centers.
L'archive ouverte pluridisciplinaire HAL, est destinée au dépôt et à la diffusion de documents scientifiques de niveau recherche, publiés ou non, émanant des établissements d'enseignement et de recherche français ou étrangers, des laboratoires publics ou privés. 


\title{
Artificial boundary conditions to compute correctors in linear elasticity
}

\author{
V. Bonnaillie-Noël, D. Brancherie ${ }^{\dagger}$ M. Dambrine ${ }^{\ddagger}$ and G. Vial ${ }^{\S}$
}

\section{Introduction}

This work is motivated by the asymptotic analysis for a boundary singular perturbation in an elliptic boundary value problem. Let $\Omega_{0}$ be a bounded domain of $\mathbb{R} \times \mathbb{R}^{+}$, with the origin $\mathbf{0}$ being a regular point on its boundary. In the following, the first coordinate axis coincides with the tangent direction of $\partial \Omega_{0}$ at point $\mathbf{0}$. We denote by $\omega$ another bounded domain containing $\mathbf{0}$. The perturbed domain $\Omega_{\varepsilon}$ is obtained from $\Omega_{0}$ by removing a rescaled version of $\omega$ at size $\varepsilon$ : $\Omega_{\varepsilon}=\Omega_{0} \backslash(\overline{\varepsilon \omega})$. The problem we focus on is the following

$$
\left\{\begin{array}{l}
-\mu \Delta \mathbf{u}_{\varepsilon}-(\lambda+\mu) \operatorname{grad} \operatorname{div} \mathbf{u}_{\varepsilon}=\mathbf{f} \text { in } \Omega_{\varepsilon} \\
\mathbf{u}_{\varepsilon}=\mathbf{u}^{d} \text { on } \Gamma_{d} \\
\boldsymbol{\sigma} \cdot \mathbf{n}=\mathbf{g} \text { on } \Gamma_{t}
\end{array}\right.
$$

where $\Gamma_{d}$ and $\Gamma_{t}$ are the Dirichlet and Neumann boundaries, respectively. It concerns Navier equations of linear elasticity. The perturbation point $\mathbf{0}$ lies on the Neumann boundary. Besides, the volumic load $\mathbf{f}$ and the traction $\mathbf{g}$ are assumed to vanish near the perturbation. Problem (1) naturally involves two scales: the size of the structure (scale 1), and the characteristic length $\varepsilon$ of the perturbation (scale $\varepsilon$ ). At scale 1 , the domain $\Omega_{\varepsilon}$ tends to $\Omega_{0}$ as $\varepsilon \rightarrow 0$, while the limit domain after rescaling (i.e. limit of $\Omega_{\varepsilon} / \varepsilon$ ) is the semi-infinite domain $\mathbf{H}_{\infty}$ defined as $\mathbf{H}_{\infty}=\left(\mathbb{R} \times \mathbb{R}^{+}\right) \backslash \bar{\omega}$. We briefly present the leading terms in the asymptotic expansion of the solution $\mathbf{u}_{\varepsilon}$ to Problem (1) obtained by following the methods presented in [5]. This description requests two variables: $x$ (slow variable) and $x / \varepsilon$ (fast variable), corresponding to scale 1 and scale $\varepsilon$, respectively. The behavior of $\mathbf{u}_{\varepsilon}$ in the fast variable relies on profiles, which are normalized functions defined in $\mathbf{H}_{\infty}$ and contributing to the expansion in variable $x / \varepsilon$. Let us introduce the profile basis $\left(\mathbf{V}_{\ell}\right)_{\ell=1,2}$ as the solutions of

$$
\left\{\begin{array}{l}
-\mu \Delta \mathbf{V}_{\ell}-(\lambda+\mu) \operatorname{grad} \operatorname{div} \mathbf{V}_{\ell}=\mathbf{0} \quad \text { in } \mathbf{H}_{\infty}, \\
\sigma\left(\mathbf{V}_{\ell}\right) \cdot \mathbf{n}=\mathbf{G}_{\ell} \quad \text { on } \partial \mathbf{H}_{\infty},
\end{array}\right.
$$

with $\mathbf{G}_{1}=\left(n_{1}, 0\right), \mathbf{G}_{2}=\left(0, n_{1}\right)$ and $\mathbf{n}=\left(n_{1}, n_{2}\right)$ the outer normal on $\partial \mathbf{H}_{\infty}$. Regularity of the solution of Navier equation has been studied, for example, in [4]. We emphasize that Problem (2) depends exclusively on the geometry. In particular, $\mathbf{V}_{\ell}$ is independent of the loading of Problem (1).

The expansion of $\mathbf{u}_{\varepsilon}$ takes the form

$$
\mathbf{u}_{\varepsilon}(\mathbf{x})=\mathbf{u}_{0}(\mathbf{x})-\varepsilon\left[\alpha_{1} \mathbf{V}_{1}\left(\frac{\mathbf{x}}{\varepsilon}\right)+\alpha_{2} \mathbf{V}_{2}\left(\frac{\mathbf{x}}{\varepsilon}\right)\right]+\mathbf{r}_{\varepsilon}(\mathbf{x})
$$

with

- $\mathbf{u}_{0}$ solves Problem (1) for $\varepsilon=0$ (i.e. is solution in the unperturbed domain $\Omega_{0}$ ),

- the coefficients $\alpha_{1}$ and $\alpha_{2}$ are the stress values associated with $\mathbf{u}_{0}$ at point $\mathbf{0}$, namely

$$
\alpha_{1}=\sigma_{11}\left(\mathbf{u}_{0}\right)(\mathbf{0}) \quad \text { and } \quad \alpha_{2}=\sigma_{12}\left(\mathbf{u}_{0}\right)(\mathbf{0}),
$$

*IRMAR, ENS Cachan Bretagne, Univ. Rennes 1, CNRS, UEB, av. Robert Schuman, F-35170 Bruz, France

${ }^{\dagger}$ Roberval, Université de Technologie de Compiègne, CNRS, F-60200 Compiègne, France

¥LMA, Université de Pau et des Pays de l'Adour, CNRS, F-64000 Pau, France

$\S$ Institut Camile Jordan, Ecole Centrale de Lyon, CNRS, F-69130 Ecully, France 
- the remainder $\mathbf{r}_{\varepsilon}$ satisfies the following estimate in the energy norm

$$
\left\|\mathbf{r}_{\varepsilon}\right\|_{\mathrm{H}^{1}\left(\Omega_{\varepsilon}\right)} \leq C \varepsilon^{2} .
$$

As regards the computation of $\mathbf{u}_{\varepsilon}$ for small values of $\varepsilon$, asymptotics expansion (3) leads to an alternative numerical strategy to adaptative mesh refinement. Indeed the knowledge of the unperturbed solution $\mathbf{u}_{0}$ and of the profiles $\mathbf{V}_{\ell}$ gives a reasonable approximation of $\mathbf{u}_{\varepsilon}$, see [1]. To approximate the profile defined by, we compute a solution of a problem posed in a bounded domain. To that end, we introduce an artificial boundary at $\partial B_{R}=\{|x|=R, R>0\}$ and we need to impose a boundary condition on this boundary in the spirit of [3]. Since, the corrector decreases at infinity, the most natural artificial condition is the Dirichlet one. However, this condition is not precise.

In this note, we present the derivation of a higher order artificial boundary condition, we explain that this condition sparks theoretical questions and finally present some numerical simulations.

\section{Seeking an artificial boundary condition}

To derive artificial boundary conditions for the linear elasticity, the domain under consideration here is the upper half plane $\mathbb{R} \times \mathbb{R}^{+}$. Since we aim at writing artificial conditions on a circle of radius $R$, it is more convenient to work with the polar coordinates and we define $\mathbf{u}=u_{r} \mathbf{e}_{r}+u_{\theta} \mathbf{e}_{\theta}$. To approximate more accurately the profile, we cancel the leading singular parts at infinity of the solution in polar coordinates.

\subsection{Computing the leading terms at infinity}

Let $L=\mu \Delta+(\lambda+\mu) \operatorname{grad} \operatorname{div}$, then

$$
\begin{aligned}
L \mathbf{u}= & \left((\lambda+2 \mu)\left[\partial_{r}^{2} u_{r}+\frac{1}{r} \partial_{r} u_{r}-\frac{1}{r^{2}} u_{r}\right]+\frac{\mu}{r^{2}} \partial_{\theta}^{2} u_{r}-\frac{\lambda+3 \mu}{r^{2}} \partial_{\theta} u_{\theta}+\frac{\lambda+\mu}{r} \partial_{r \theta}^{2} u_{\theta}\right) \mathbf{e}_{r} \\
& +\left(\mu\left[\partial_{r}^{2} u_{\theta}+\frac{1}{r} \partial_{r} u_{\theta}-\frac{1}{r^{2}} u_{\theta}\right]+\frac{\lambda+2 \mu}{r^{2}} \partial_{\theta}^{2} u_{\theta}+\frac{\lambda+\mu}{r} \partial_{r \theta}^{2} u_{r}+\frac{\lambda+3 \mu}{r^{2}} \partial_{\theta} u_{r}\right) \mathbf{e}_{\theta} .
\end{aligned}
$$

In polar coordinates, the stress tensor takes the form:

$$
\sigma(\mathbf{u})=\left[\begin{array}{cc}
(\lambda+2 \mu) \partial_{r} u_{r}+\frac{\lambda}{r}\left(u_{r}+\partial_{\theta} u_{\theta}\right) & \mu\left(\frac{1}{r}\left(\partial_{\theta} u_{r}-u_{\theta}\right)+\partial_{r} u_{\theta}\right) \\
\mu\left(\frac{1}{r}\left(\partial_{\theta} u_{r}-u_{\theta}\right)+\partial_{r} u_{\theta}\right) & (\lambda+2 \mu) \frac{1}{r}\left(\partial_{\theta} u_{\theta}+u_{r}\right)+\lambda \partial_{r} u_{r}
\end{array}\right] .
$$

We seek the displacement under the form $\mathbf{u}=r^{s}\left[\varphi_{r}(\theta), \varphi_{\theta}(\theta)\right]^{T}$. Consequently, we obtain

$$
\begin{aligned}
L \mathbf{u} & =r^{s-2}\left[\begin{array}{cc}
\mu \varphi_{r}^{\prime \prime}+(\lambda+2 \mu)\left(s^{2}-1\right) \varphi_{r}+[(\lambda+\mu) s-(\lambda+3 \mu)] \varphi_{\theta}^{\prime} \\
(\lambda+2 \mu) \varphi_{\theta}^{\prime \prime}+\mu\left(s^{2}-1\right) \varphi_{\theta}+[(\lambda+\mu) s+(\lambda+3 \mu)] \varphi_{r}^{\prime}
\end{array}\right], \\
\sigma(\mathbf{u}) & =r^{s-1}\left[\begin{array}{cc}
\lambda \varphi_{\theta}^{\prime}+((\lambda+2 \mu) s+\lambda) \varphi_{r} & \mu\left(\varphi_{r}^{\prime}+(s-1) \varphi_{\theta}\right) \\
\mu\left(\varphi_{r}^{\prime}+(s-1) \varphi_{\theta}\right) & (\lambda+2 \mu) \varphi_{\theta}^{\prime}+(\lambda s+(\lambda+2 \mu)) \varphi_{r}
\end{array}\right] .
\end{aligned}
$$

Using (6), we reduce the second order system $L \mathbf{u}=0$ to a bigger system of first order: Define $\psi_{r}=\varphi_{r}^{\prime}$ and $\psi_{\theta}=\varphi_{\theta}^{\prime}$, and $\mathbf{U}=\left(\varphi_{r}, \varphi_{\theta}, \psi_{r}, \psi_{\theta}\right)^{T}$, then

$$
\mathbf{U}^{\prime}=A \mathbf{U},
$$

with

$$
A=\left[\begin{array}{cccc}
0 & 0 & 1 & 0 \\
0 & 0 & 0 & 1 \\
\frac{(\lambda+2 \mu)\left(1-s^{2}\right)}{\mu} & 0 & 0 & \frac{(\lambda+3 \mu)-(\lambda+\mu) s}{\mu} \\
0 & \frac{\mu\left(1-s^{2}\right)}{\lambda+2 \mu} & -\frac{(\lambda+3 \mu)+(\lambda+\mu) s}{\lambda+2 \mu} & 0
\end{array}\right]
$$


The eigenvalues of $A$ are $\pm i(s \pm 1)$. Hence, the polar functions $\varphi_{r}, \varphi_{\theta}$ belong to the space generated by $\cos ((s \pm 1) \theta), \sin ((s \pm 1) \theta)$. We look for coefficients $A_{r}, B_{r}, A_{\theta}, B_{\theta}$ so that

$$
\begin{aligned}
& \varphi_{r}=A_{r} \cos ((s-1) \theta)+B_{r} \sin ((s-1) \theta)+C_{r} \cos ((s+1) \theta)+D_{r} \sin ((s+1) \theta), \\
& \varphi_{\theta}=A_{\theta} \cos ((s-1) \theta)+B_{\theta} \sin ((s-1) \theta)+C_{\theta} \cos ((s+1) \theta)+D_{\theta} \sin ((s+1) \theta) .
\end{aligned}
$$

Writing $L \mathbf{u}=0$ gives:

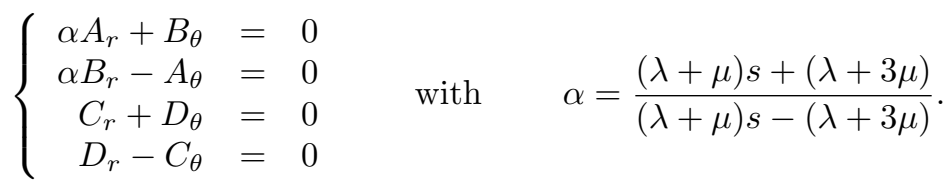

Consequently, the functions $\varphi_{r}$ and $\varphi_{\theta}$ satisfy:

$$
\begin{aligned}
\varphi_{r} & =A_{r} \cos ((s-1) \theta)+B_{r} \sin ((s-1) \theta)+C_{r} \cos ((s+1) \theta)+D_{r} \sin ((s+1) \theta), \\
\varphi_{\theta} & =\alpha B_{r} \cos ((s-1) \theta)-\alpha A_{r} \sin ((s-1) \theta)+D_{r} \cos ((s+1) \theta)-C_{r} \sin ((s+1) \theta) .
\end{aligned}
$$

The boundary conditions read: $\sigma(\mathbf{u}) \cdot \mathbf{e}_{\theta}=\mathbf{0}$ for $\Theta=0, \pi$, that is to say

$$
\left\{\begin{aligned}
\varphi_{r}^{\prime}(\Theta)+(s-1) \varphi_{\theta}(\Theta) & =0 \\
(\lambda+2 \mu) \varphi_{\theta}^{\prime}(\Theta)+((\lambda+2 \mu) s+\lambda) \varphi_{r}(\Theta) & =0
\end{aligned}\right.
$$

Boundary conditions at $\theta=0$ reads

$$
\left\{\begin{aligned}
(s-1) B_{r}+(s+1) D_{r}+(s-1)\left(A_{\theta}+C_{\theta}\right) & =0 \\
(\lambda+2 \mu)\left((s-1) B_{\theta}+(s+1) D_{\theta}\right)+(\lambda s+\lambda+2 \mu)\left(A_{r}+C_{r}\right) & =0 .
\end{aligned}\right.
$$

Boundary conditions at $\theta=\pi$ reads

$$
\left\{\begin{array}{rrr}
\sin s \pi\left[(s-1)\left(A_{r}-B_{\theta}-D_{\theta}\right)+(s+1) C_{r}\right]-\cos s \pi\left[(s-1)\left(A_{\theta}+B_{r}+C_{\theta}\right)+(s+1) D_{r}\right] & = & 0 \\
\sin s \pi\left((\lambda+2 \mu)\left[(s-1)\left(A_{\theta}+(s+1) C_{\theta}\right]-(\lambda+2 \mu+\lambda s)\left(B_{r}+D_{r}\right)\right)\right. & \\
-\cos s \pi\left((\lambda+2 \mu)\left[(s-1) B_{\theta}+(s+1) D_{\theta}\right]+(\lambda s+\lambda+2 \mu)\left(A_{r}+C_{r}\right)\right) & =0
\end{array}\right.
$$

Using (8), we deduce that the coefficients $A_{r}, B_{r}, C_{r}, D_{r}$ have to satisfied

$$
M\left(A_{r}, B_{r}, C_{r}, D_{r}\right)^{T}=0,
$$

with

$$
M=\left[\begin{array}{cccc}
0 & \gamma & 0 & 2 s \\
\beta & 0 & -2 \mu s & 0 \\
\gamma \sin s \pi & -\gamma \cos s \pi & 2 s \sin s \pi & -2 s \cos s \pi \\
-\beta \cos s \pi & -\beta \sin s \pi & 2 \mu s \cos s \pi & 2 \mu s \sin s \pi
\end{array}\right]
$$

and

$$
\beta=(1+\alpha)(\lambda+2 \mu)+2(\lambda-\alpha(\lambda+2 \mu)), \quad \gamma=(s-1)(1+\alpha) .
$$

We compute

$$
\operatorname{det} M=-4(\sin s \pi)^{2}(\mu \gamma+\beta)^{2} .
$$

Therefore, $s \mapsto \operatorname{det} M$ cancels only for $s \in \mathbb{Z}$ and for $s$ such that $\mu \gamma+\beta=0$ that is to say

$$
s=\frac{\mu-\lambda+\sqrt{\lambda^{2}+6 \lambda \mu+25 \mu^{2}}}{2 \mu}>2 \quad \text { and } \quad s=\frac{\mu-\lambda-\sqrt{\lambda^{2}+6 \lambda \mu+25 \mu^{2}}}{2 \mu}<-2 .
$$

Then the leading term is obtained for $s=-1$. For $s=-1$, we have

$$
\alpha=-\frac{\mu}{\lambda+2 \mu}, \quad \beta=0, \quad \gamma=-\frac{2(\lambda+\mu)}{\lambda+2 \mu} .
$$

In this case, system $M\left(A_{r}, B_{r}, C_{r}, D_{r}\right)^{T}=0$ and (8) read

$$
\gamma B_{r}-2 D_{r}=0, \quad 2 \mu C_{r}=0, \quad C_{\theta}=D_{r} .
$$

Consequently, setting $A=A_{r}$ and $B=-B_{r}$ in (9), the functions $\varphi_{r}$ and $\varphi_{\theta}$ take the form

$$
\left\{\begin{array}{l}
\varphi_{r}=A \cos 2 \theta+B \sin 2 \theta \\
\varphi_{\theta}=\frac{\mu}{\lambda+2 \mu}(B \cos 2 \theta-A \sin 2 \theta)+B \frac{\lambda+\mu}{\lambda+2 \mu}
\end{array}\right.
$$




\subsection{Using the singular profiles to obtain a transparent boundary condi- tion for the half plane}

We go back to the expression for $\sigma(\mathbf{u}) \cdot \mathbf{e}_{r}$ deduced from $(7)$. When $s=-1$, we have

$$
\sigma(\mathbf{u}) \cdot \mathbf{e}_{r}=r^{-2}\left[\begin{array}{c}
\lambda \varphi_{\theta}^{\prime}-2 \mu \varphi_{r} \\
\mu\left(\varphi_{r}^{\prime}-2 \varphi_{\theta}\right)
\end{array}\right]=r^{-1}\left[\begin{array}{cc}
-\frac{4 \mu(\lambda+\mu)}{\lambda+2 \mu} & 0 \\
-\frac{2 \mu(\lambda+\mu) \sin 2 \theta}{\mu+(\lambda+\mu) \cos 2 \theta} & \frac{2 \mu(\lambda+\mu)(1-\cos 2 \theta)}{\mu+(\lambda+\mu) \cos 2 \theta}
\end{array}\right] \mathbf{u} .
$$

This means that the relation between $\mathbf{u}$ and $\sigma(\mathbf{u}) \cdot \mathbf{n}$ on $\partial B_{R}$ has variable (in the $\theta$ variable) coefficients, this fact makes the interpretation of this computation more difficult. Hence, we search a relation with the second order tangential derivative of the displacement: If such a relationship exists, the associated bilinear form still remains symmetric. Notice that

$$
\varphi_{r}^{\prime}=-\frac{\lambda+2 \mu}{2 \mu} \varphi_{\theta}^{\prime \prime}
$$

we can also write

$$
\sigma(\mathbf{u}) \cdot \mathbf{e}_{r}=r^{-2}\left[\begin{array}{c}
4 \mu(\lambda+\mu) \\
-\frac{\lambda+2 \mu}{\lambda+} \varphi_{r} \\
\mu \varphi_{r}^{\prime}-2 \mu \varphi_{\theta}
\end{array}\right]=\frac{1}{R}\left[\begin{array}{c}
-\frac{4 \mu(\lambda+\mu)}{\lambda+2 \mu} u_{r} \\
-2 \mu u_{\theta}-\frac{\lambda+2 \mu}{2} \partial_{\theta}^{2} u_{\theta}
\end{array}\right] .
$$

For the elasticity problem, we observe that the leading decreasing profile at infinity satisfies the following inequality on the circle of radius $R$ :

$$
\sigma(\mathbf{u}) \cdot \mathbf{n}+\frac{2 \mu}{R}\left[\begin{array}{cc}
\frac{2(\lambda+\mu)}{\lambda+2 \mu} & 0 \\
0 & 1
\end{array}\right] \mathbf{u}+\frac{\lambda+2 \mu}{2 R}\left[\begin{array}{ll}
0 & 0 \\
0 & 1
\end{array}\right] \partial_{\theta}^{2} \mathbf{u}=0 .
$$

Lamé's coefficients are linked to Young's modulus and Poisson's coefficient throught the relations:

$$
\lambda=\frac{\nu E}{(1+\nu)(1-2 \nu)}, \quad \mu=\frac{E}{2(1+\nu)} \quad \text { with } \quad E>0, \quad-1<\nu<0.5 .
$$

So that, this boundary condition of Ventcel's type rewrites on $\partial B_{R} \cap \mathbb{R} \times \mathbb{R}^{+}$:

$$
\sigma(\mathbf{u}) \cdot \mathbf{n}+\frac{1}{R} \frac{E}{1+\nu}\left[\begin{array}{cc}
\frac{1}{1-\nu} & 0 \\
0 & 1
\end{array}\right] \mathbf{u}+\frac{1}{R} \frac{E(1-\nu)}{2(1+\nu)(1-2 \nu)}\left[\begin{array}{ll}
0 & 0 \\
0 & 1
\end{array}\right] \partial_{\theta}^{2} \mathbf{u}=0 .
$$

The main difficulty here is that the coefficient $\frac{E(1-\nu)}{2(1+\nu)(1-2 \nu)}$ is positive; meaning that the variational formulation of the boundary value problem for the profiles $\mathbf{V}_{\ell}$

$$
\left\{\begin{array}{l}
-\mu \Delta \mathbf{V}_{\ell}-(\lambda+\mu) \operatorname{grad} \operatorname{div} \mathbf{V}_{\ell}=\mathbf{0} \quad \text { in } B_{R} \cap \mathbf{H}_{\infty}, \\
\sigma\left(\mathbf{V}_{\ell}\right) \cdot \mathbf{n}=\mathbf{G}_{\ell} \quad \text { on } \partial \mathbf{H}_{\infty} \cap B_{R}, \\
\sigma\left(\mathbf{V}_{\ell}\right) \cdot \mathbf{n}+\frac{1}{R} \frac{E}{1+\nu}\left[\begin{array}{cc}
\frac{1}{1-\nu} & 0 \\
0 & 1
\end{array}\right] \mathbf{V}_{\ell}+\frac{E(1-\nu)}{2(1+\nu)(1-2 \nu) R}\left[\begin{array}{ll}
0 & 0 \\
0 & 1
\end{array}\right] \partial_{\theta}^{2} \mathbf{V}_{\ell}=0 \quad \text { on } \partial B_{R} \cap \mathbf{H}_{\infty},
\end{array}\right.
$$

is not coercive. Therefore, the question of existence and uniqueness of the solution to (12) is open. For the model scalar case of

$$
\left\{\begin{array}{l}
-\Delta V=0 \text { in } \Omega \\
\partial_{n} V+\alpha V+\beta \Delta_{\Gamma} V=G \text { on } \partial \Omega
\end{array}\right.
$$

with $\beta>0$ and $\Delta_{\Gamma}$ denotes the Laplace-Beltrami operator on $\partial \Omega$, it has been shown in [2] that, for a fixed $R$, the boundary value problem has a unique solution if $\alpha / \beta$ avoid a countable set of values, and moreover, that it has a unique solution for $R$ large enough. We expect similar results for the elasticity case and we work in that direction. 


\section{Numerical results}

In order to investigate Problem (12) from a numerical point of view, we have considered the test situation where the domain $\omega$ is the top half of a ball: $\mathbf{H}_{\infty}=\mathbb{R} \times \mathbb{R}^{+} \backslash B(0,1)$. We have successively considered the following two questions:

(i) resolubility of the Ventcel-type boundary condition (11),

(ii) order of precision of this condition as an artificial boundary condition for Problem (2).

For the first question, we consider the norm of the inverse operator $\left(L_{R}\right)^{-1}$ corresponding to Problem (12):

$$
L_{R}: \mathbf{V}_{\ell} \mapsto \mathbf{G}_{\ell}
$$

In Figure 1, we show the evolution of this norm with respect to the radius $R$. The computation have been performed with the finite element library MÉLINA (see [6]), the domain (a ring) has been meshed into 128 quadrangular elements of degree 8, and a $\mathbb{Q}_{10}$ interpolation is used for the finite element method.

Figure 1 shows some values of $R$ for which the resolvent is unbounded, but theses values remain close to 1 . It seems that Problem (12) is uniquely solvable for $R$ sufficiently large (which is the result shown for the scalar model problem).
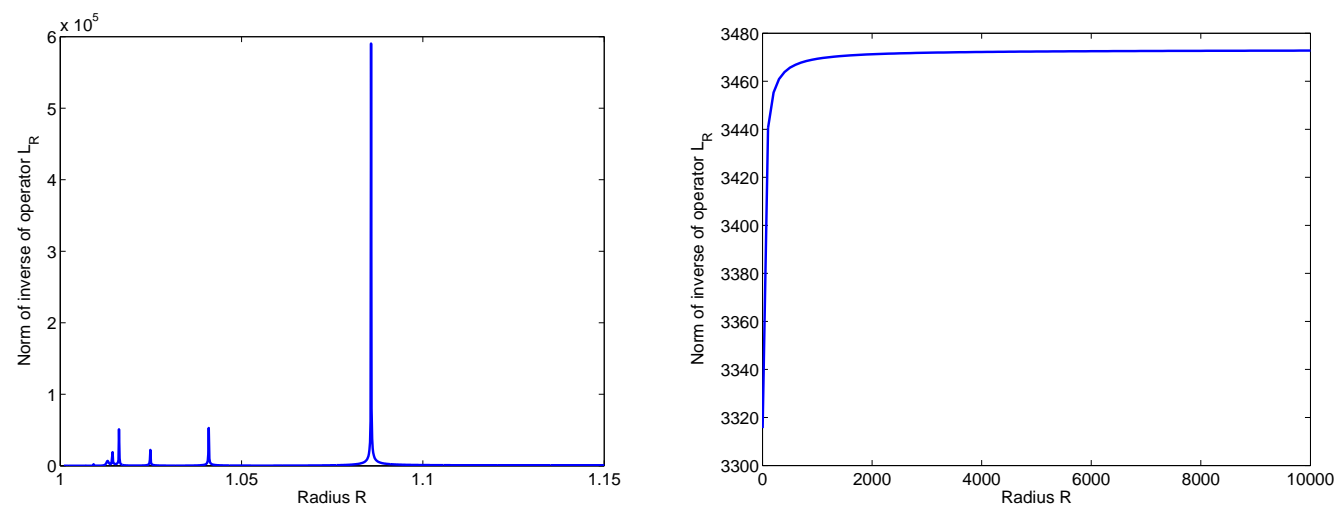

Figure 1: Norm of $\left(L_{R}\right)^{-1}$ with respect to $R$.

For question (ii), we consider Problem (12) where the data $\mathbf{G}_{\ell}$ is chosen so that the solution $\mathbf{V}_{\ell}$ is the sum of two dual singular solutions:

$$
\mathbf{V}_{\ell}=\mathbf{s}^{1}+\mathbf{s}^{2},
$$

where $\mathbf{s}^{1}$ is given by (2.1) and $\mathbf{s}^{2}$ has the form

$$
\mathbf{s}^{2}(r, \theta)=r^{-2}\left[\varphi_{r}^{2}(\theta) \mathbf{e}_{r}+\varphi_{\theta}^{2}(\theta) \mathbf{e}_{\theta}\right]
$$

with

$$
\begin{gathered}
\varphi_{r}^{2}(\theta)=A^{\prime} \cos (3 \theta)-B^{\prime} \sin (3 \theta)+\frac{3 \alpha \lambda-\lambda-4 \mu}{4 \mu} A^{\prime} \cos \theta+\frac{3}{4}(1+\alpha) B^{\prime} \sin \theta, \\
\varphi_{\theta}^{2}(\theta)=\alpha B^{\prime} \cos (3 \theta)+\alpha A^{\prime} \cos (3 \theta)-\frac{3}{4}(1+\alpha) B^{\prime} \cos \theta+\frac{3 \alpha \lambda-\lambda-4 \mu}{4 \mu} A^{\prime} \sin \theta,
\end{gathered}
$$

and

$$
\alpha=\frac{-\lambda+\mu}{-3 \lambda-5 \mu} .
$$

With this choice, the function $\mathbf{V}_{\ell}$ solves Problem (2) in the infinite domain $\mathbf{H}_{\infty}$. In Figure 2, we show the $\mathrm{H}^{1}$-norm of the difference between the exact solution of (2) and its approximation with artificial boundary conditions (Dirichlet or Ventcel). It turns out that - as expected - the Dirichlet condition is of order 1 and the Ventcel condition of order 2 .

Remark 1 Writing the variational formulation of (12) makes appear ponctual terms which need a specific treatment for the numerical approach with the finite element method. 


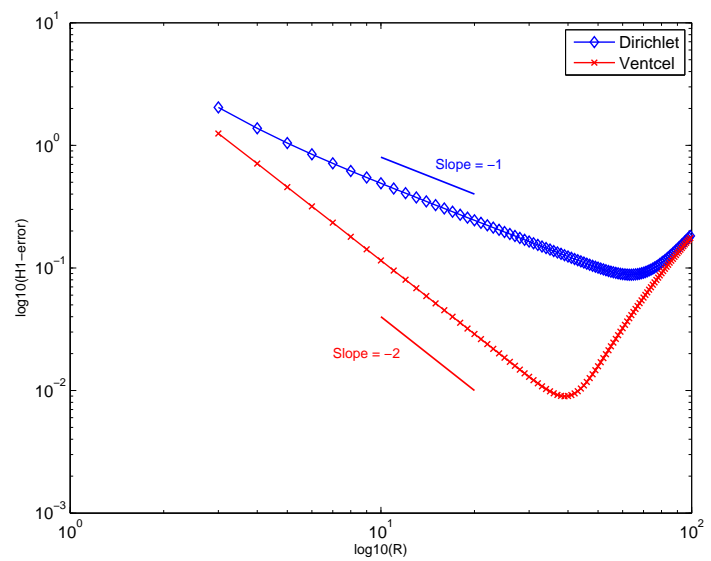

Figure 2: Precision of the artificial conditions with respect to $R$.

\section{References}

[1] V. Bonnaillie-Noël, D. Brancherie, M. Dambrine, F. Hérau, S. Tordeux and G. Vial, Multiscale expansion and numerical approximation for surface defects, ESAIM Proc., To appear (2011)

[2] V. Bonnaillie-Noël, M. Dambrine, F. Hérau, and G. Vial, On generalized Ventcel's type boundary conditions for Laplace operator in a bounded domain, SIAM Journal on Mathematical Analysis 42 (2010), no. 2, 931-945.

[3] D. Givoli. Nonreflecting boundary conditions. J. Comput. Phys. 94(1) (1991) 129.

[4] P. Grisvard, Problèmes aux limites dans les polygônes. Mode d'emploi, EDF Bull. Direction Études Rech. Sér. C Math. Inform. 1 (1986), no. 3, 21-59.

[5] V. Maz'ya, S. Nazarov and B. Plamenevskij, Asymptotic theory of elliptic boundary value problems in singularly perturbed domains. Vol. I-II, Operator Theory: Advances and Applications, Vol 111, 112, Birkhäuser Verlag, 2000

[6] D. Martin, http://perso.univ-rennes1.fr/daniel.martin/melina (2008). 